\title{
Comprehensive Identification of Key Genes Involved in Development of Diabetes Mellitus-Related Atherogenesis Using Weighted Gene Correlation Network Analysis
}

\author{
Qi Huang ${ }^{1,2+}$, Guoxiong Deng ${ }^{1,2 \dagger}$, Rongguo Wei ${ }^{3,4 \dagger}$, Qiaoye Wang ${ }^{5,6}$, Donghua Zou ${ }^{5,6 *}$ and \\ Jinru Wei ${ }^{1,2 *}$
}

OPEN ACCESS

Edited by:

Seitaro Nomura

The University of Tokyo, Japan

Reviewed by:

Ville-Petteri Makinen,

South Australian Health and Medical Research Institute (SAHMRI), Australia

Paras Kumar Mishra,

University of Nebraska Medical

Center, United States

*Correspondence:

Donghua Zou

danvor0922@hotmail.com

Jinru Wei

weijinru@stu.gxmu.edu.cn

tThese authors have contributed equally to this work

Specialty section:

This article was submitted to Cardiovascular Genetics and Systems

Medicine,

a section of the journal

Frontiers in Cardiovascular Medicine

Received: 06 July 2020

Accepted: 15 September 2020

Published: 28 October 2020

Citation:

Huang $Q$, Deng $G$, Wei $R$, Wang $Q$,

Zou D and Wei J (2020)

Comprehensive Identification of Key

Genes Involved in Development of

Diabetes Mellitus-Related Atherogenesis Using Weighted Gene

Correlation Network Analysis.

Front. Cardiovasc. Med. 7:580573.

doi: 10.3389/fCVm.2020.580573
${ }^{1}$ Department of Cardiology, The Fifth Affiliated Hospital of Guangxi Medical University, Nanning, China, ${ }^{2}$ Department of Cardiology, The First People's Hospital of Nanning, Nanning, China, ${ }^{3}$ Department of Clinical Laboratory, The Fifth Affiliated Hospital of Guangxi Medical University, Nanning, China, ${ }^{4}$ Department of Clinical Laboratory, The First People's Hospital of Nanning, Nanning, China, ${ }^{5}$ Department of Neurology, The Fifth Affiliated Hospital of Guangxi Medical University, Nanning, China, ${ }^{6}$ Department of Neurology, The First People's Hospital of Nanning, Nanning, China

Coronary heart disease (CHD) is common in patients with diabetes mellitus (DM), however, the relevant mechanism remains elusive. The whole blood gene expression profiles of healthy control, patients with DM, patients with DM and CHD (DMCHD) were used to performed weight gene correlation network analysis (WGCNA) to identify the gene modules associated with DM-related atherogenesis. The candidate module was significantly involved in immune- and $T$ cell activity-related biological process. GSEA results suggested that lysosome and apoptosis were enriched in DM and DMCHD samples. The protein-protein-KEGG pathway network may reveal the potential transcriptional regulatory network involving in DM-related atherosclerosis. Nineteen genes (RTKN, DCP1B, PDZD4, CACNA2D2, TSEN54, PVRIG, PLEKHF1, NKG7, ZAP70, NUDCD3, SLAMF6, CCDC107, NAG6, ZDHHC14, EOMES, VIL2, WDR54, DMAP1, and PMPCA) were considered as DM-related atherogenesis genes (DRAGs). The Gene Set Variation Analysis (GSVA) score of the DRAG set gradually increased in the control, DM and DMCHD. ROC curve analysis showed that ZAP70, TSEN54, and PLEKHF1 may be potential blood circulation biomarkers for DMCHD in patients with DM. In conclusion, we identified nineteen hallmark genes involving in DM-related atherogenesis and constructed a potential transcriptional regulatory network involving in DM-related atherosclerosis.

Keywords: diabetes mellitus, coronary heart disease, hallmark gene set, WGCNA, atherogenesis

\section{INTRODUCTION}

Diabetes mellitus (DM) is a metabolic disorder of the endocrine system, clinically characterized by hyperglycemia as well as alterations in lipids, carbohydrates, and protein metabolism $(1,2)$. DM can be divided into type 1 and type 2 , of which more than $90 \%$ of cases are type $2(3,4)$. Thus, only type $2 \mathrm{DM}$ was focused in our present study. The increasing prevalence of type $2 \mathrm{DM}$ means that 430 million people are expected to suffer the condition by $2030(5,6)$. DM renders patients more 
susceptible to arterial atherosclerosis, which causes cardiovascular disease, especially coronary heart disease (CHD). $\mathrm{CHD}$ is the most serious complication of DM and a frequent cause of death among DM patients (7). Thus, elucidating why $\mathrm{DM}$ increases risk of CHD is of great importance.

Several studies have suggested that the presence of hypertension, dyslipidemia, and/or obesity in most patients with DM may help explain the increased incidence of $\mathrm{CHD}$, since these factors also increase CHD risk in individuals without DM (8-14). Given that DM is multifactorial and quite heterogeneous (15), a comprehensive examination is required to understand key mechanisms driving development of CHD in patients.

To address this issue, we attempted to identify hub genes or gene sets involved in DM-associated CHD (DMCHD). Using weighted correlation network analysis (WGCNA), we identified 19 DM-related atherogenesis genes (DRAGs). Based on proteinprotein interaction and functional enrichment analysis, we constructed a protein-protein pathway network to uncover the potential transcriptional regulatory network involved in DMrelated atherosclerosis.

\section{MATERIALS AND METHODS}

\section{Data Processing}

We downloaded gene expression profiles from the Gene Expression Omnibus (https://www.ncbi.nlm.nih.gov/) (16) to analyze genetic differences between DM and DMCHD. We extracted the data set from the human whole blood gene expression profile of GSE34198 based on GPL6947 (17), which included 33 healthy control samples, 34 CHD samples, 15 DM samples and 15 DMCHD samples. In GSE34198, CHD patients refers to patients who eventually develop acute myocardial infarction and DM refers to type 2 diabetes. The clinical information including sex, age, body mass index and smoking status was provided in the Supplementary Table 1. GSE90074 (18) based on GPL6480 was as a validation set and contained human whole blood mononuclear cells gene expression profiles from 33 healthy control samples, $55 \mathrm{CHD}$ samples, $17 \mathrm{DM}$ samples, and 38 DMCHD samples. The normalize Between Arrays function in the limma package (19) was used to normalize the gene expression profiles. Probes that corresponded to multiple genes were removed. If a gene was detected using multiple probes, the expression level of the gene was calculated by averaging the expression from all the probes.

\section{Screening Differently Expressed Genes (DEGs) in DM and DMCHD Compared to Healthy Control}

To identify the genes involving in DM-related atherogenesis, the DEGs in DM and DMCHD compared to healthy control were screened using limma package. A gene with $\mathrm{P}$ adjusted by false discovery rate $<0.05$ was considered significantly differently expressed.

\section{WGCNA}

It is well-known that $\mathrm{DM}$ increases risk of $\mathrm{CHD}$, thus, healthy control, DM, and DMCHD were considered as different stages of DM-related atherosclerosis. According to WGCNA official website (https://horvath.genetics.ucla.edu/ html/CoexpressionNetwork/Rpackages/WGCNA/), this method is suitable for identifying gene modules with the certain phenotype (DM-related atherosclerosis). DM increases the risk of CHD is considered the result of multiple molecules working together. WGCNA was used to identify the related gene modules (each module contain multiple molecules). Thus, WGCNA (20) was selected in the present study. The DEGs were subjected to perform a weighted gene expression network in GSE34198 using the WGCNA package (20) in R version 3.5 (https://www. r-project.org/). Candidate power (1 to 20) was used to test the average connectivity degrees of different modules and degrees of independence. If the degree of independence was $>0.85$, the power value was selected. The hclust function was used to cluster samples and check for outliers. Subsequently, a heat map was constructed to correlate gene modules with phenotype and statistical significance. High correlation meant that the genes of the corresponding module tended to be highly correlated with development of CHD in DM patients. In the present study, the module with the most significant positive correlation to DM-related atherogenesis was considered the candidate module.

\section{Functional Enrichment Analysis of Gene Modules}

In order to explore the signaling pathways and biological characteristics of related genes in phenotype-related modules, we conducted Gene Ontology (GO) and Kyoto Encyclopedia of Genes and Genomes (KEGG) pathway enrichment analysis. The clusterProfiler package (21) in $\mathrm{R}$ were applied to these functional enrichment analysis. In addition, a biological processes network was constructed using the ClueGO plug-in (22) in Cytoscape (23). GSEA software (https://www.gseamsigdb.org/gsea/downloads.jsp) was used to analyze gene set enrichment (24) based on the reference gene sets "c5.bp.v7.0. symbols.gmt" and "c2.cp.kegg.v7.0.symbols.gmt" (25). $P<0.05$ was considered significant.

\section{Construction of Protein-Protein KEGG Pathway Networks}

The STRING database (https://string-db.org/) (26) was used to extract protein-protein interactions between candidate module genes. Cytoscape was used to construct a protein-protein network based on KEGG pathways involved in DM-related atherogenesis.

\section{Identification of DM-Related Atherogenesis Genes (DRAGs) and Assessment of Predictive Potential}

In the candidate module based on WGCNA, genes with significance $(\mathrm{GS})>0.2$ and module membership $(\mathrm{MM})>0.7$ were considered as DM-related atherogenesis genes (DRAGs). The GSVA package (27) was used to perform gene set variation analysis (GSVA) to score individual samples against the DRAG 


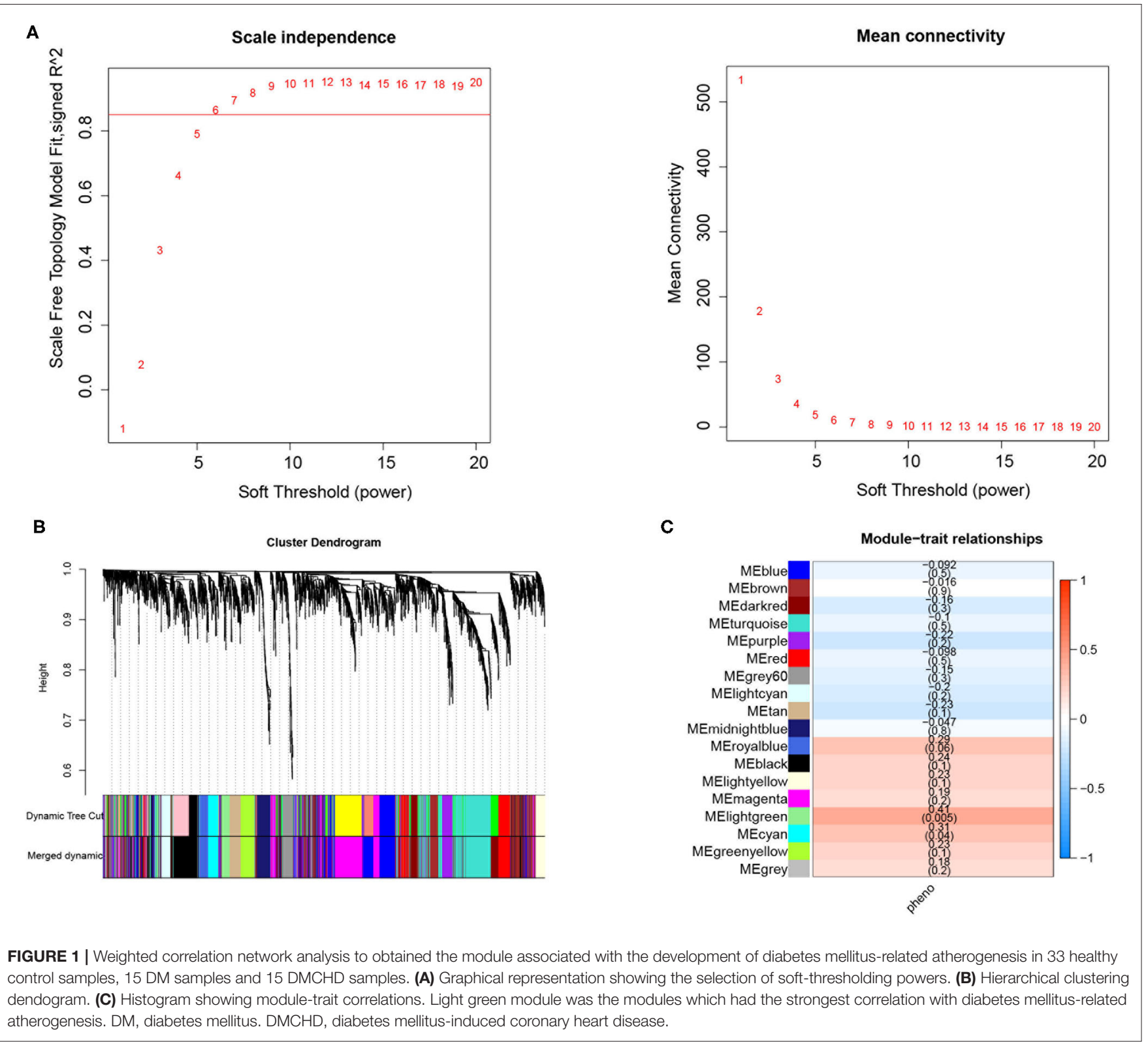

set, and the DM-related atherogenesis GSVA score was obtained for each sample. The ability of the DM-related atherogenesis GSVA score to predict DMCHD was evaluated using receiver operating characteristic (ROC) curve analysis with the $p R O C$ package (28). The aberrant expression of DRAGs were validated using the GSE90074 dataset. DRAGs with high predictive value were considered as biomarkers of DMCHD.

\section{Cell Subpopulation Analysis}

The host's response to DM and DMCHD may also be reflected in blood cell subsets. The webtool xCell (29) (https://xcell.ucsf. edu/) was used to perform cell type enrichment analysis from gene expression data. Use analysis of variance to compare the cell subpopulations abundance in control, DM, and DMCHD.

\section{RESULTS}

\section{Determining DRAG Modules}

In the current study, a total of 2,442 DEGs were screened with 1,029 up-regulated and 1,413 down-regulated in in DM and DMCHD compared to healthy control. The expression profiles of the 2,442 DEGs were subjected to WGCNA. In the WGCNA, according to the results of the scale-free fit index and average connectivity, the soft-thresholding was determined as six (Figure 1A). Based on hierarchical clustering, the genes were divided into 18 modules (Figure 1B). The light green module was involved in immune- and $\mathrm{T}$ cell activity-related biological processes, and displayed the most significant positive correlation between modules and DMrelated atherogenesis $(r=0.41, p=0.005)$ (Figure 1C). 


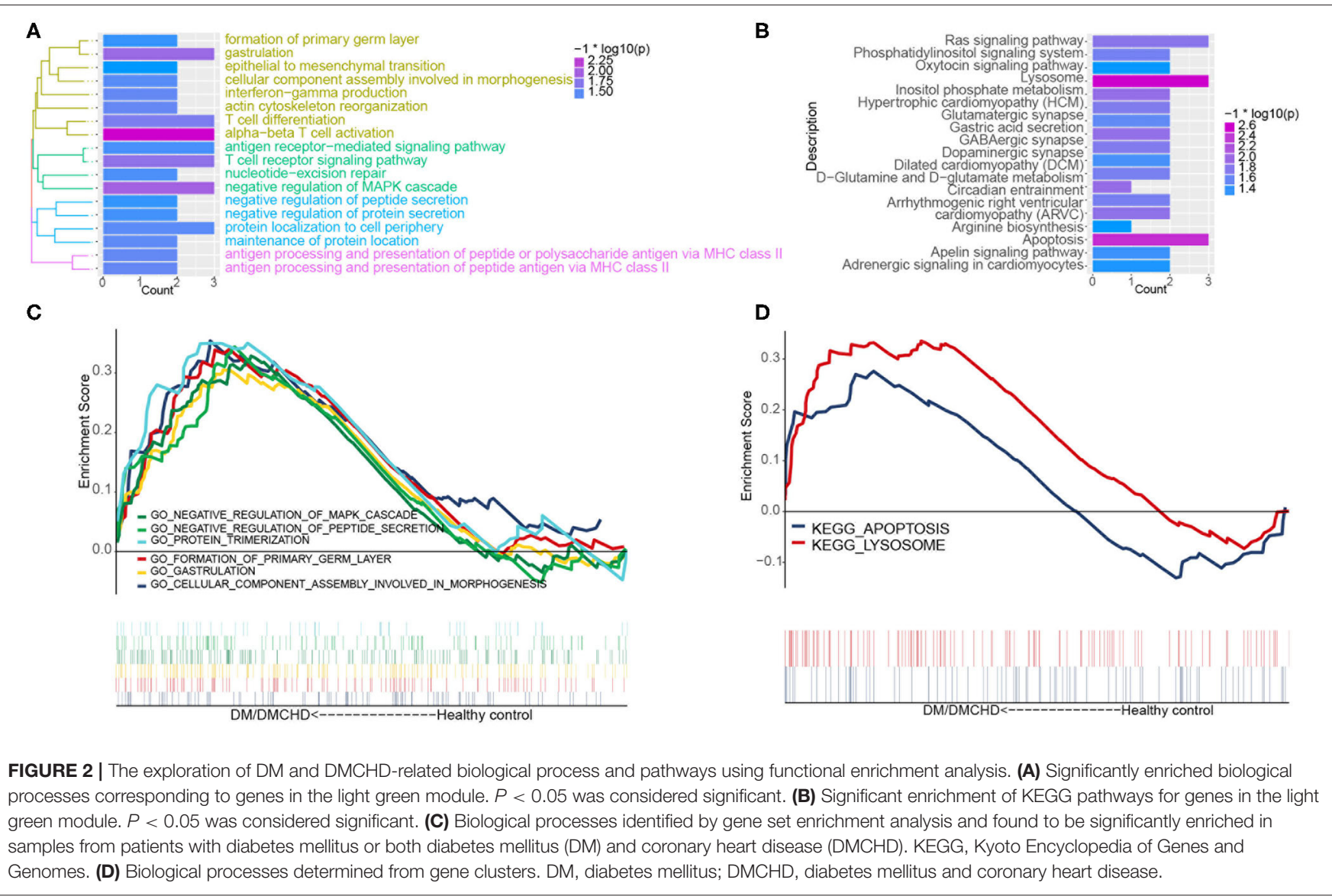

It was therefore considered as the candidate module in subsequent analyses.

\section{Biological Functions Involving the Candidate Module}

The light green module contained genes significantly involved in biological processes related to cytoskeletal anchoring at plasma membrane, protein localization to cell cortex and protein deneddylation (Figure 2A). Genes within the module were associated with autophagy, phosphatidylinositol biosynthetic processes and $\mathrm{G}$ protein-coupled receptor signaling pathways (Supplementary Figure 1). Moreover, the light green module was significantly involved in pathways involving lysosomes, apoptosis, Ras signaling pathways and inositol phosphate metabolism (Figure 2B). DM and DMCHD samples showed enrichment in gastrulation and protein trimerization (Figure 2C), as well as lysosome and apoptosis pathways (Figure 2D).

\section{Interaction Between Proteins Encoded by DM and CHD}

A protein-protein KEGG pathway network was constructed to provide a potential mechanism explaining how DM increases risk of CHD. The network included 51 module-related genes and 18 KEGG pathways (Figure 3A). Previous studies proposed that arginine biosynthesis, GABAergic synapse and gastric acid secretion pathways overlap between DM and CHD (30-32). In this study, we elucidated the following pathways potentially involved in DMCHD: adrenergic signaling in cardiomyocytes, apelin signaling, circadian entrainment, oxytocin signaling pathway, apoptosis and Ras signaling (Figure 3B). The arenergic signaling in cardiomyocytes was activated by $\mathrm{Ca}^{2+}$ signaling, cell phosphorylation was mediated by CAMKII and CACNA2D2. In addition, we found that CNGT2 could affect cell proliferation. The apelin signaling pathway might cause vasodilatation and cardiovascular development. The $\mathrm{G}$ protein-coupled receptor APJ and its cognate ligand what apelin were widely expressed throughout the human body. They implicated in different key physiological processes such as angiogenesis, cardiovascular functions and energy metabolism regulation. Moreover, they also involved in the development and progression of different pathologies including diabetes, obesity and cardiovascular disease (33). In the circadian entrainment, presynaptic RGC neuron secreted L-glutamate and PACAP. Furthermore, PAC1 could activate c-Fos by CNGT2. In the oxytocin signaling pathway, CACNA2D2 and CALM3 could indirectly affect cardiovascular system and renal epithelial cell. Besides, the oxytocin signaling pathway was also activated by $\mathrm{Ca} 2+$ signaling. In the apoptosis, sphingosine and lysosomotropic detergents 
A

$$
\begin{gathered}
\text { Dopaminergic synapse } \\
\text { Dilated cardiomyopathy (DCM) GABAergic synapse }
\end{gathered}
$$

D-Glutamine and D-glutamate

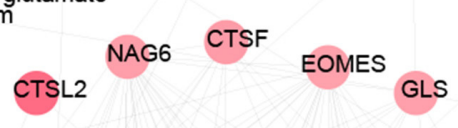

Circadian entrainment NUDCD3

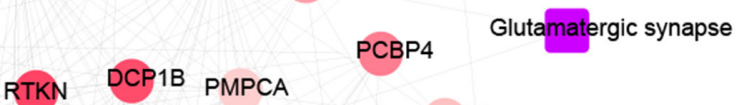

RTKN DCP1B PMPCA

Arrhythmogenic right ventricular

DMAP1

Gastric acid secretion
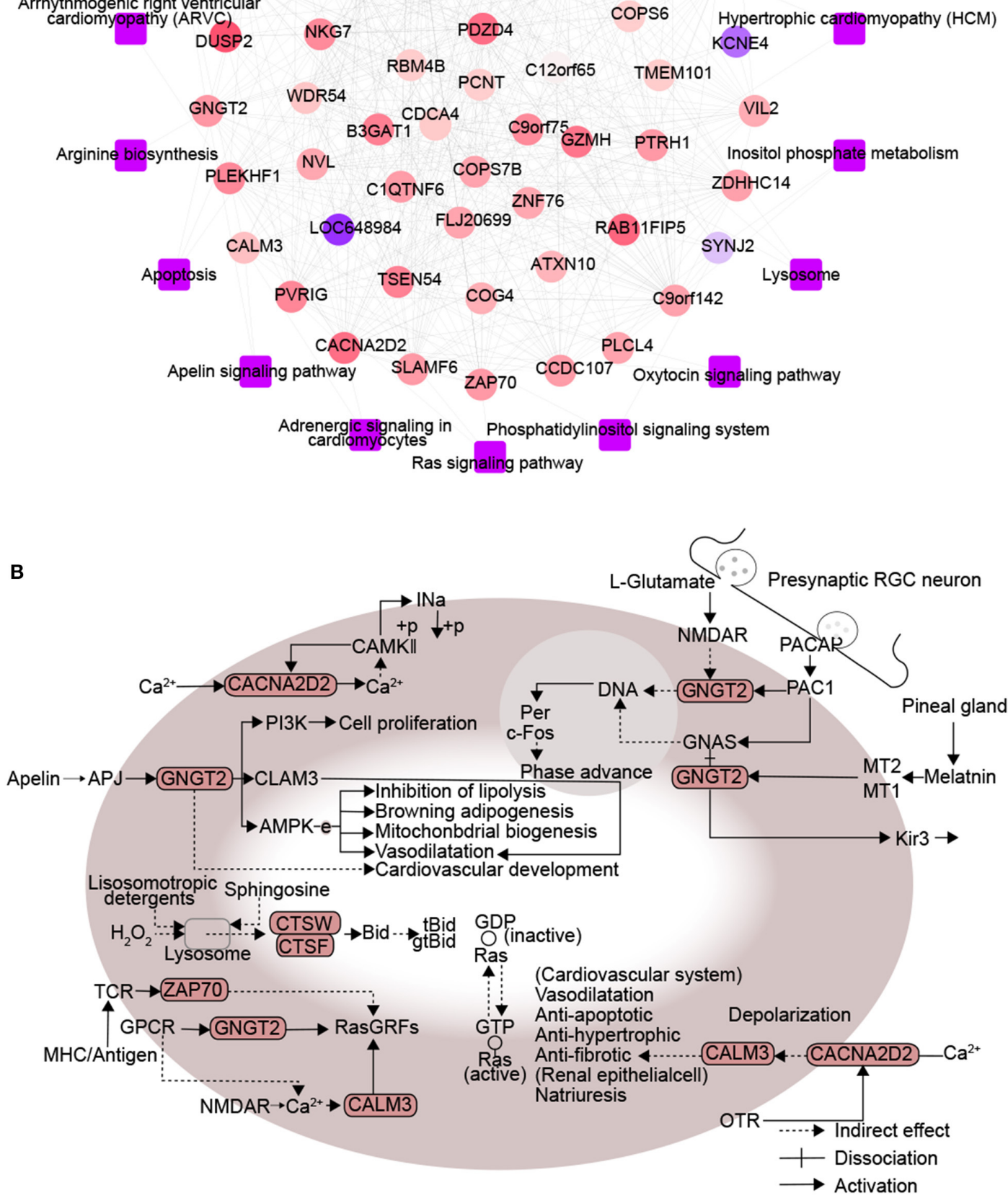

FIGURE 3 | Protein interactions associated with signaling pathways enriched in diabetes mellitus-associated coronary heart disease. (A) Network diagram of protein-protein-KEGG pathway related to DMCAD. The ellipse for the module gene. The round rectangle for the KEGG pathway. The color of gene nodes represents MM, and the color at the edge of gene nodes represents modules. (B) CALM3, ZAP70, GNGT2, CACNA2D2, CTSF, and CTSW-related KEGG pathways. DMCHD, diabetes mellitus-induced coronary heart disease. DRAG, DM-related atherogenesis gene. MM, module membership. 
and $\mathrm{H}_{2} \mathrm{O} 2$ could indirectly affect lysosome. CTSW and CTSF could be indirectly affected by lysosome, thereby affecting cell apoptosis. In the Ras signaling pathway, CNGT2 and CALM3 could activate GDP-Ras inactive to GTP-Ras active process by RasGRFs. Moreover, ZAP70 could also indirectly affect the process by RasGRFs.

\section{The Expression of DRAGs and ROC Analysis of DM-Related Atherogenesis GSVA Score}

We identified the following genes from the light green module as significant DRAG candidates: RTKN, DCP1B, PDZD4, CACNA2D2, TSEN54, PVRIG, PLEKHF1, NKG7, ZAP70, NUDCD3, SLAMF6, CCDC107, NAG6, ZDHHC14, EOMES, VIL2, WDR54, DMAP1, and PMPCA. Their expression progressively increased in healthy control, DM and DMCHD (Figure 4A), the differential expression of these genes in three different groups healthy control, DM, DMCHD might be the reason which lead to DM patients with coronary heart disease, but whether this is the cause of this result needs further exploration. ROC curves showed that the DM-related atherogenesis GSVA score can predict DMCHD, with an area under the ROC curve of 0.743 (Figure 4B).

\section{Validating Aberrant Expression of DRAGs}

To validate the DRAGs we identified, we examined their expression levels in the GSE90074 dataset. We confirmed that ZAP70, PLEKHF1, and TSEN54 expression significantly progressively increased in healthy control, DM and DMCHD (Figures 5A-D), these genes were considered to be the progression genes of DM complicated with CHD. ROC curve analysis suggested that all three genes may be potential biomarkers of CHD development in DM (Figure 5E).

\section{Various Cell Types Vary in Control, DM and DMCHD}

After estimating and comparing the cell subpopulation abundance, we found the various cell types vary in control, DM and DMCHD. B cells and Macrophages (M2) showed an increasing trend, while eosinophils, common lymphoid progenitor cell (CLP), and granulocyte macrophage progenitor (GMP) showed a downward trend. Osteoblast, Gamma delta T cells (Tgd cells), and endothelial cells are also different in control, DM and DMCHD (Figure 6).

\section{DISCUSSION}

DM is a syndrome characterized by chronic hyperglycemia accompanied by disorder of lipid and protein metabolism. The pathogenesis of diabetes includes insulin resistance and insulin secretion disorders of islet beta cells. CHD is a kind of heart disease caused by atherosclerotic lesion of coronary artery, which leads to stenosis or obstruction of vascular lumen and myocardial ischemia, hypoxia or necrosis. DM and CHD are common chronic diseases (34). People with DM have a much higher incidence of CHD because of dyslipidemia and metabolic disorders, particularly in the formation of glycated proteins, causing inflammation and artery stiffening as well as dysregulating hormones, immune responses and dendritic cell activity (35). However, the genetic changes that underlie development of CHD in patients with DM is not fully understood. In the present study, we performed WGCNA in a whole-blood gene expression profile to screen for gene modules involved in the pathogenesis of DM-related atherogenesis. We found that a light green module, a cluster of immune system genes, had the strongest correlation with emergence of CHD in DM. Gene sets associated with apoptosis and lysosomes were particularly enriched in DM and DMCHD samples. These findings are consistent with a known link between apoptosis and CHD (36) and between lysosome activity and DMCHD (37).

A protein-protein KEGG pathway network was constructed to help reveal the molecular mechanism of DMCHD. Among the six pathways identified, previous work has already implicated apelin signaling (38), apoptosis (39), and Ras signaling (40) in DMCHD. In addition, our study suggests that adrenergic signaling in cardiomyocytes, circadian entrainment and oxytocin signaling pathway are also related to DMCHD. CACNA2D2 and GNGT2 play important roles in regulating these pathways. Expression of CACNA2D2 in the adrenergic signaling pathway elevates intracellular $\mathrm{Ca}^{2+}$ in cardiomyocytes, enhancing myocardial contractility (41), and more generally changes in gene expression have been associated with $\mathrm{Ca}^{2+}$ mobilization in CHD (42). Regulation of GNGT2 affects circadian entrainment, in which c-Fos may be activated to regulate apoptosis, although this is controversial $(43,44)$. The activation of c-Fos in gestational diabetes suggests a link to glucose metabolism, which may therefore be linked to DMCHD, which requires further study. Notably, oxytocin promotes glucose metabolism in cultured cardiomyocytes from newborn and adult rats (45). Furthermore, it can directly improve pancreatic functions (46), presumably by increasing insulin early (47). Oxytocin signaling pathway is also activated by $\mathrm{Ca}^{2+}$ signaling in a CACNA2D2-dependent manner. These data support targeting oxytocin in DM patients in order to prevent CHD.

$\mathrm{DM}$ is a complicated, polygenic disease that exerts pleotropic effects that may increase risk of other diseases, such as CHD. Therefore, it is necessary to identify hallmark genes that explain potential mechanism of DM-related atherogenesis. We identified 19 genes in the light green module as DRAGs (RTKN, DCP1B, PDZD4, CACNA2D2, TSEN54, PVRIG, PLEKHF1, NKG7, ZAP70, NUDCD3, SLAMF6, CCDC107, NAG6, ZDHHC14, EOMES, VIL2, WDR54, DMAP1, and PMPCA), the differential expression of these genes in three different groups healthy control, DM, DMCHD might be the reason which lead to DM patients with coronary heart disease, but whether this is the cause of this result needs further exploration. We found that the DM-related atherogenesis GSVA score progressively increased in healthy control, DM and DMCHD samples. ROC analysis showed that DM-related atherogenesis GSVA score may have predictive potential for identifying genes responsible for DMCHD development. Our work suggests that the DRAGs ZAP70, PLEKHF1, and TSEN54 may be biomarkers for CHD 
A GSE34198

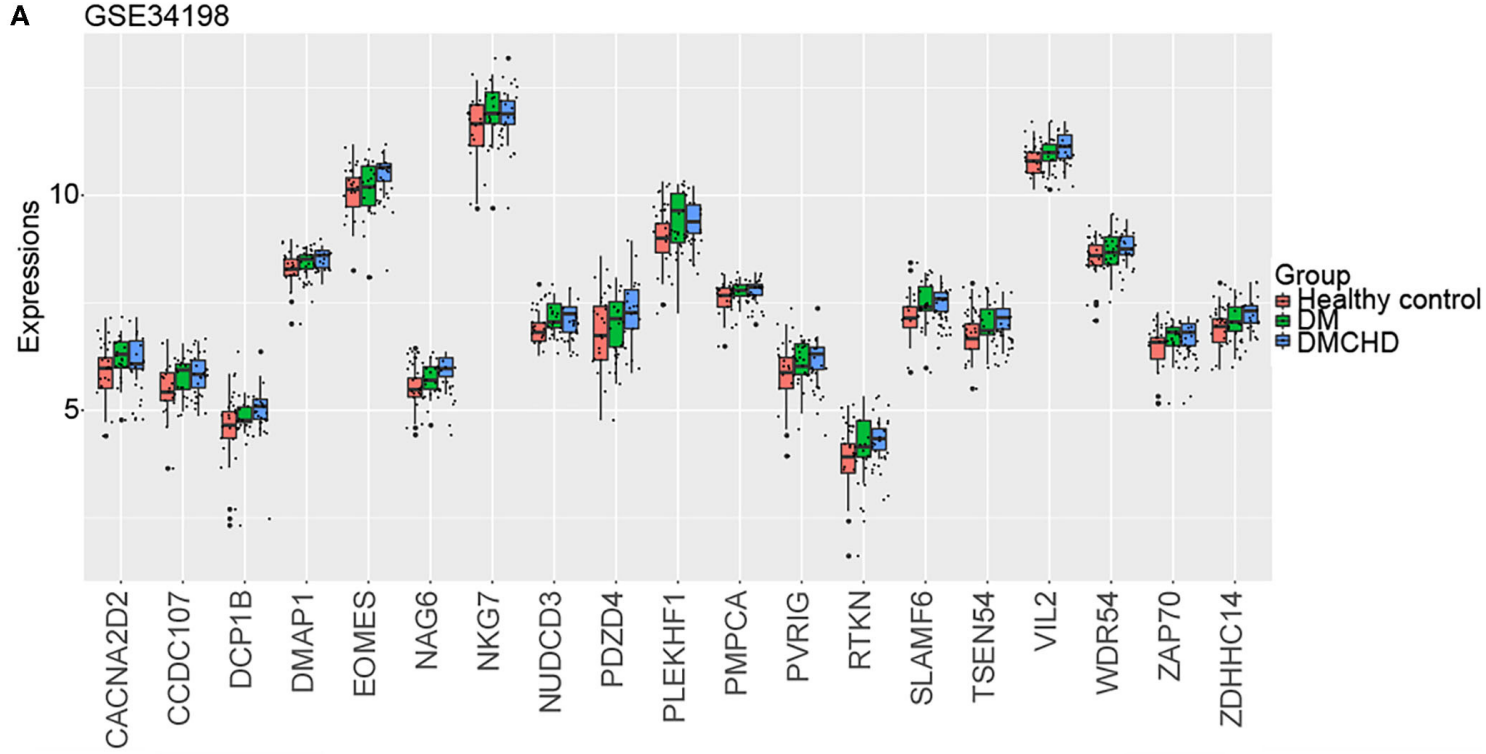

B

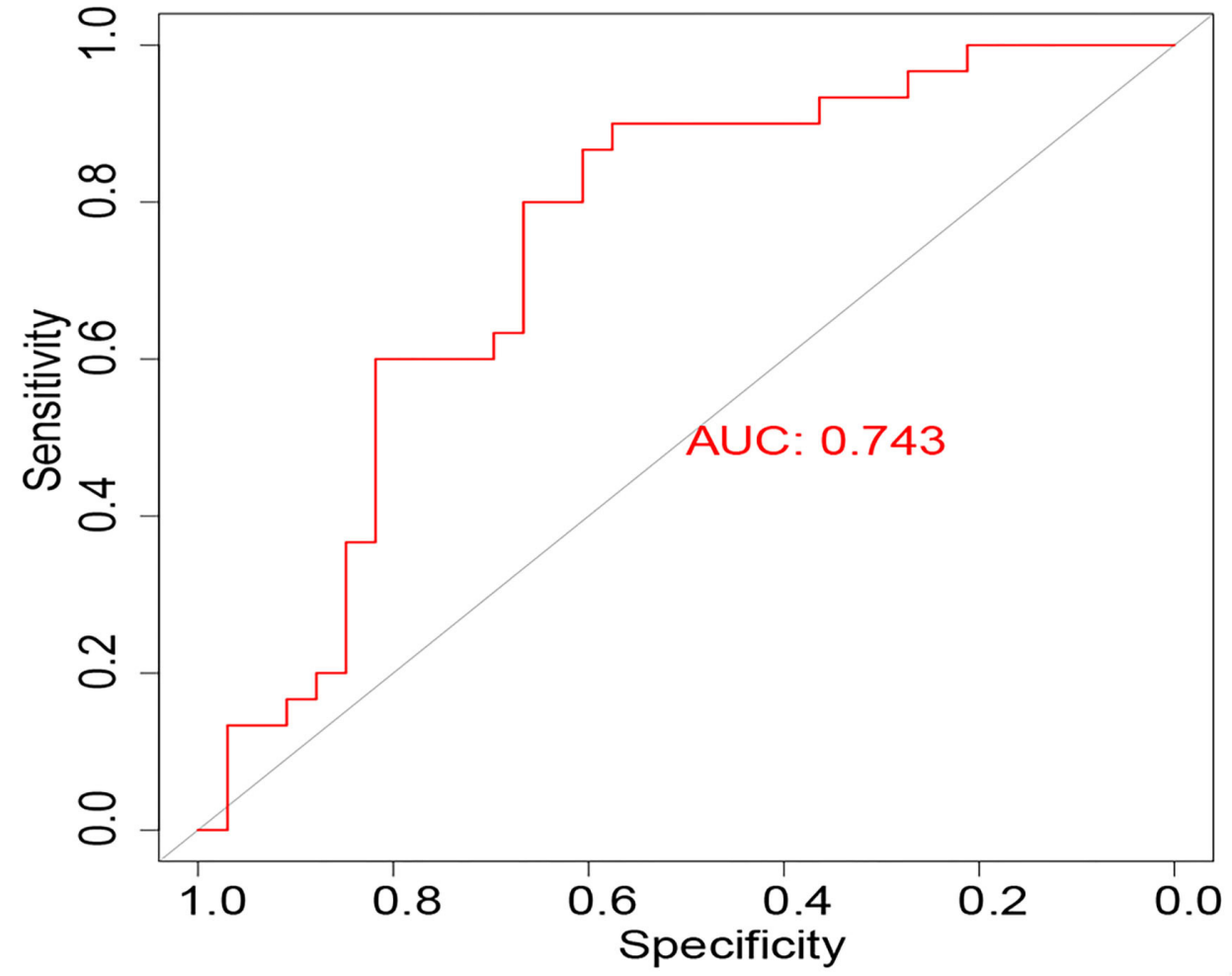

FIGURE 4 | Expression of diabetes mellitus-related atherogenesis genes (DRAGs) and their ability to predict diabetes mellitus-associated coronary heart disease (DMCHD). (A) The expression level of 19 DRAGs (genes with significance (GS) $>0.2$ and module membership (MM) $>0.7$ were considered as DRAGs) in tissue from healthy individuals and from patients with diabetes mellitus (DM) or DMCHD. (B) Receiver operating characteristic curve analysis of DM-related atherogenesis GSVA score for predicting DMCHD.

in DM patients. In addition, we also found some types of cells in whole blood showed upward or downward trend in control, DM and DMCHD. However, the abundance of the cell subsets will be affected by many factors, and whether it can be used as a specific marker of DM or DMCHD still needs further exploration.

There are several notable limitations in our study. First, we took a bioinformatics approach to mine potential molecular 


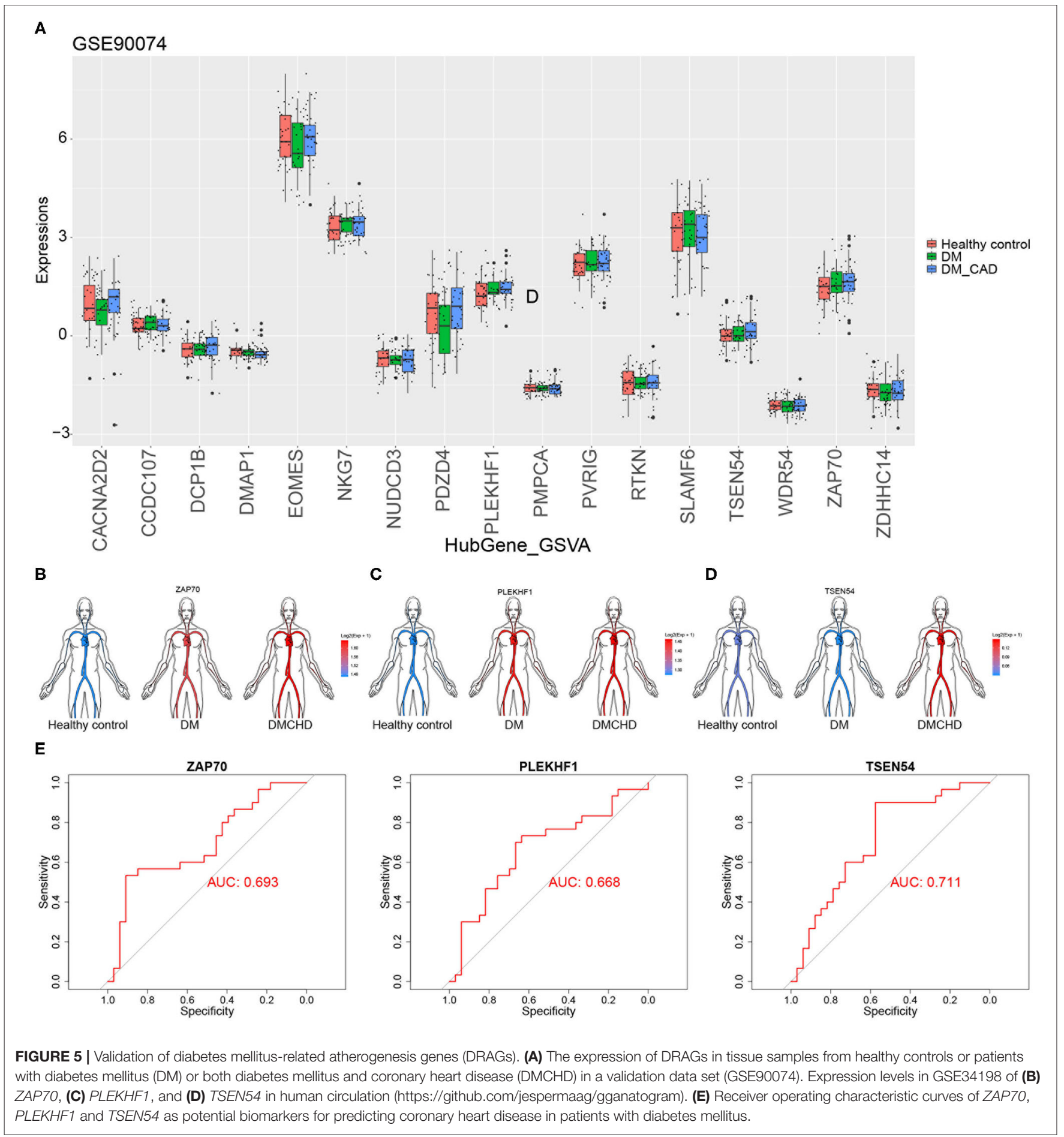

mechanisms causing DMCHD. Our findings will need to be followed up with mechanistic studies. Second, using GSVA to assess presence of genetic risk factors in individual patients is cost-prohibitive and therefore not applicable in clinics yet. Third, only one dataset was used for validation. Whether ZAP70, PLEKHF1, and TSEN54 are true biomarkers for DMCHD requires further examination in additional, larger data sets. In addition, it is worth noting that the whole blood gene expression profiles were used in the present study. We also found various cell types vary in control, DM and DMCHD, however, we can not elaborate on the interactions between cells in the DM-related atherogenesis in the present study.

In conclusion, we identified 19 hallmark genes of DMrelated atherogenesis, which might be the biomarkers for 


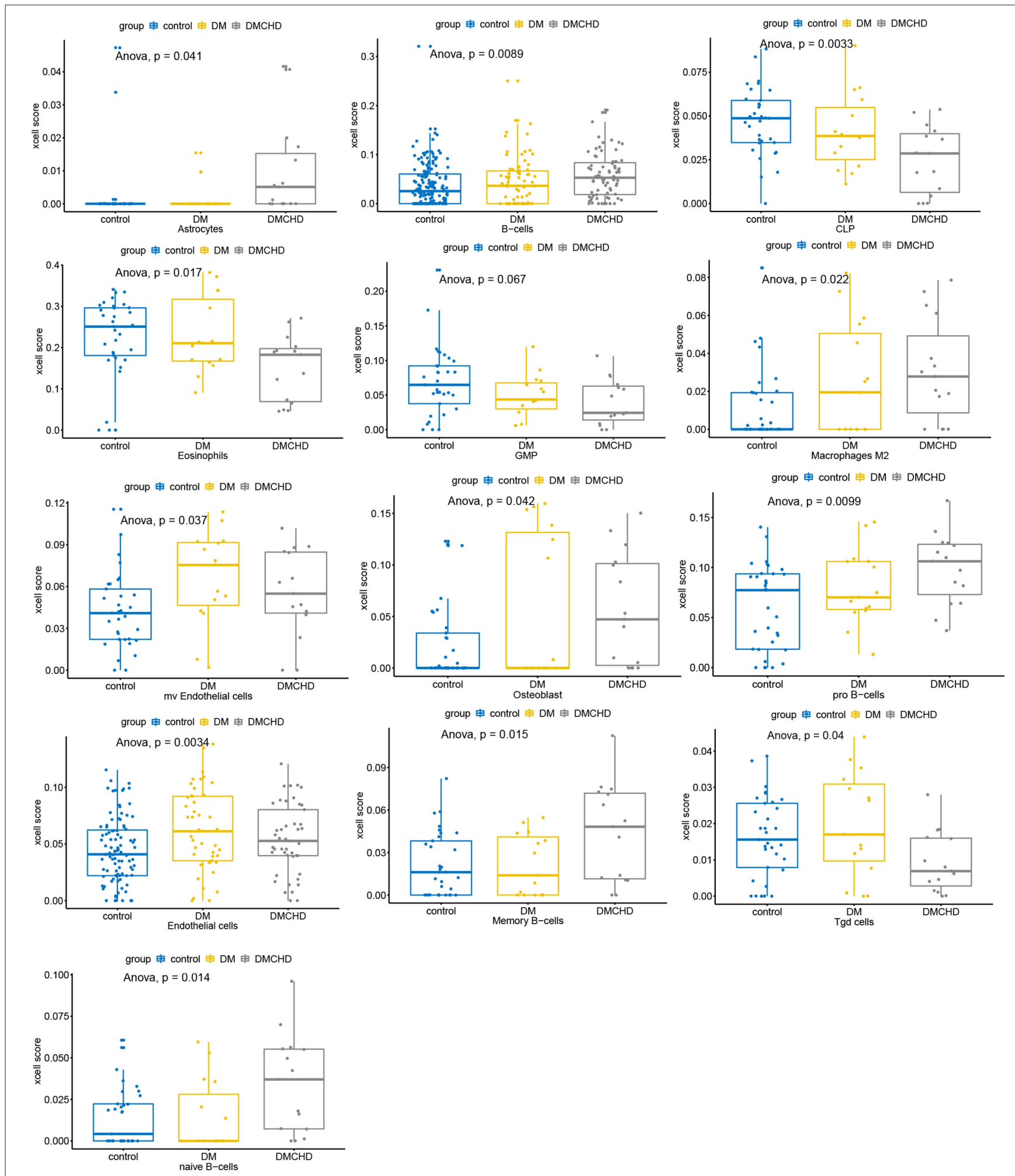

FIGURE 6 | Various cell types vary in control, DM and DMCHD. DM, diabetes mellitus, DMCHD, diabetes mellitus and coronary heart disease, CLP, common lymphoid progenitor cell; GMP, granulocyte macrophage progenitor; Tgd cells, gamma delta T cells; mv endothelial cells, mitral valve endothelial cells. Anova, analysis of variance. 
identifying DM and CHD. The DM-related atherogenesis GSVA score may predict susceptibility of DM patients to developing CHD. Our protein-protein KEGG pathway network may reveal molecular mechanisms underlying DMCHD development. These results provide numerous leads for future studies of CHD risk factors in DM, and may provide a theoretical basis for the treatment of DM and CHD patients.

\section{DATA AVAILABILITY STATEMENT}

The datasets generated for this study can be found in online repositories. The names of the repository/repositories and accession number(s) can be found in the article/Supplementary Material.

\section{AUTHOR CONTRIBUTIONS}

All authors participated in the design, interpretation of the studies, analysis of the data, and review of the manuscript. $\mathrm{QH}$ and GD conducted the experiments. RW supplied critical reagents. DZ wrote the manuscript. JW contributed to critically

\section{REFERENCES}

1. Ramachandran S, Rajasekaran A, Manisenthilkumar KT. Investigation of hypoglycemic, hypolipidemic and antioxidant activities of aqueous extract of Terminalia paniculata bark in diabetic rats. Asian Pac J Trop Biomed. (2012) 2:262-8. doi: 10.1016/S2221-1691(12)60020-3

2. Rashidi AA, Mirhashemi SM, Taghizadeh M, Sarkhail P. Iranian medicinal plants for diabetes mellitus: a systematic review. Pak J Biol Sci. (2013) 16:40111. doi: 10.3923/pjbs.2013.401.411

3. Yang SH, Dou KF, Song WJ. Prevalence of diabetes among men and women in China. N Engl J Med. (2010) 362:2425-6. doi: 10.1056/NEJMoa0908292

4. Patil P, Mandal S, Tomar SK, Anand S. Food protein-derived bioactive peptides in management of type 2 diabetes. Eur J Nutr. (2015) 54:86380. doi: 10.1007/s00394-015-0974-2

5. Chang MH, Valdez R, Ned RM, Liu T, Yang Q, Yesupriya A, et al. Influence of familial risk on diabetes risk-reducing behaviors among U.S. adults without diabetes. Diabetes Care. (2011) 34:2393-9. doi: 10.2337/dc11-0876

6. Mustafa SB, Mehmood Z, Akhter N, Kauser A, Hussain I, Rashid A, et al. Review-medicinal plants and management of diabetes mellitus: a review. Pak J Pharm Sci. (2016) 29:1885-91.

7. Ullah F, Afridi AK, Rahim F, Ur Rahman S, Ashfaq M. Efficacy of $5 \mathrm{Mg}$ and $10 \mathrm{Mg}$ rosuvastatin in Type-2 diabetes mellitus with hypercholesteroalemia. $J$ Ayub Med Coll Abbottabad. (2015) 27:564-8.

8. Defronzo RA. Insulin resistance, hyperinsulinemia, and coronary artery disease: a complex metabolic web. J Cardiovasc Pharmacol. (1992) 20(Suppl. 11):S1-16. doi: 10.1097/00005344-199200111-00002

9. Solymoss BC, Marcil M, Chaour M, Gilfix BM, Poitras AM, Campeau L. Fasting hyperinsulinism, insulin resistance syndrome, and coronary artery disease in men and women. Am J Cardiol. (1995) 76:11526. doi: 10.1016/S0002-9149(99)80326-9

10. Despres JP, Lamarche B, Mauriege P, Cantin B, Dagenais GR, Moorjani S, et al. Hyperinsulinemia as an independent risk factor for ischemic heart disease. $N$ Engl J Med. (1996) 334:952-7. doi: 10.1056/NEJM199604113341504

11. Grossman E, Messerli FH. Diabetic and hypertensive heart disease. Ann Intern Med. (1996) 125:304-10. doi: 10.7326/0003-4819-125-4-199608150-00009

12. Roh E, Ko SH, Kwon HS, Kim NH, Kim JH, Kim CS, et al. Prevalence and management of dyslipidemia in Korea: Korea national health and nutrition revise the manuscript. All authors contributed to the article and approved the submitted version.

\section{FUNDING}

This study was supported by the Project of Nanning Scientific Research and Technology Development Plan (20193252), the Nanning Qingxiu District Science and Technology Plan Project (2018029), the High-Level Medical Expert Training Program of Guangxi 139 Plan Funding (G201903049), and Guangxi medical and health key discipline construction project. This study was also sponsored by the Nanning Excellent Young Scientist Program and the Guangxi Beibu Gulf Economic Zone Major Talent Program (RC20190103).

\section{SUPPLEMENTARY MATERIAL}

The Supplementary Material for this article can be found online at: https://www.frontiersin.org/articles/10.3389/fcvm. 2020.580573/full\#supplementary-material

Supplementary Figure 1 | Biological process enrichment analysis using ClueGO plugin for genes in the light green module. examination survey during 1998 to 2010. Diabetes Metab J. (2013) 37:43349. doi: 10.4093/dmj.2013.37.6.433

13. Kim CS, Ko SH, Kwon HS, Kim NH, Kim JH, Lim S, et al. Prevalence, awareness, and management of obesity in Korea: data from the Korea national health and nutrition examination survey (1998-2011). Diabetes Metab J. (2014) 38:35-43. doi: 10.4093/dmj.2014.38.1.35

14. Ko SH, Kwon HS, Kim DJ, Kim JH, Kim NH, Kim CS, et al. Higher prevalence and awareness, but lower control rate of hypertension in patients with diabetes than general population: the fifth korean national health and nutrition examination survey in 2011. Diabetes Metab J. (2014) 38:517. doi: 10.4093/dmj.2014.38.1.51

15. Zimmet $P$, Alberti KG, Shaw J. Global and societal implications of the diabetes epidemic. Nature. (2001) 414:782-7. doi: 10.1038/414782a

16. Barrett T, Wilhite SE, Ledoux P, Evangelista C, Kim IF, Tomashevsky M, et al. NCBI GEO: archive for functional genomics data sets-update. Nucleic Acids Res. (2013) 41:D991-5. doi: 10.1093/nar/gks1193

17. Jiang YX, Jing LD, Jia YH. Clinical characteristics and risk factors of left ventricular thrombus after acute myocardial infarction: a matched casecontrol study. Chin Med J. (2015) 128:2415-9. doi: 10.4103/0366-6999. 164869

18. Ravi S, Schuck RN, Hilliard E, Lee CR, Dai X, Lenhart K, et al. Clinical evidence supports a protective role for CXCL5 in coronary artery disease. Am J Pathol. (2017) 187:2895-911. doi: 10.1016/j.ajpath.2017. 08.006

19. Ritchie ME, Phipson B, Wu D, Hu Y, Law CW, Shi W, et al. limma powers differential expression analyses for RNA-sequencing and microarray studies. Nucleic Acids Res. (2015) 43:e47. doi: 10.1093/nar/gkv007

20. Langfelder P, Horvath S. WGCNA: an $\mathrm{R}$ package for weighted correlation network analysis. BMC Bioinformatics. (2008) 9:559. doi: 10.1186/1471-2105-9-559

21. Yu G, Wang LG, Han Y, He QY. clusterProfiler: an R package for comparing biological themes among gene clusters. OMICS. (2012) 16:284-7. doi: 10.1089/ omi.2011.0118

22. Bindea G, Mlecnik B, Hackl H, Charoentong P, Tosolini M, Kirilovsky A, et al. ClueGO: a cytoscape plug-in to decipher functionally grouped gene ontology and pathway annotation networks. Bioinformatics. (2009) 25:10913. doi: 10.1093/bioinformatics/btp101 
23. Kohl M, Wiese S, Warscheid B. Cytoscape: software for visualization and analysis of biological networks. Methods Mol Biol. (2011) 696:291303. doi: 10.1007/978-1-60761-987-1_18

24. Subramanian A, Tamayo P, Mootha VK, Mukherjee S, Ebert BL, Gillette MA, et al. Gene set enrichment analysis: a knowledge-based approach for interpreting genome-wide expression profiles. Proc Natl Acad Sci USA. (2005) 102:15545-50. doi: 10.1073/pnas.0506580102

25. Liberzon A, Birger C, Thorvaldsdottir H, Ghandi M, Mesirov JP, Tamayo P. The molecular signatures database (MSigDB) hallmark gene set collection. Cell Syst. (2015) 1:417-25. doi: 10.1016/j.cels.2015.12.004

26. Szklarczyk D, Morris JH, Cook H, Kuhn M, Wyder S, Simonovic M, et al. The STRING database in 2017: quality-controlled protein-protein association networks, made broadly accessible. Nucleic Acids Res. (2017) 45:D3628. doi: 10.1093/nar/gkw937

27. Herranz Amo F, Rivero Sanchez E, Verdu Tartajo F, Hernandez Fernandez C, Diez Cordero JM. [Cysts of the seminal vesicles: apropos of a case]. Actas Urol Esp. (1987) 11:210-3

28. Robin X, Turck N, Hainard A, Tiberti N, Lisacek F, Sanchez JC, et al. pROC: an open-source package for $\mathrm{R}$ and $\mathrm{S}+$ to analyze and compare ROC curves. BMC Bioinformatics. (2011) 12:77. doi: 10.1186/1471-2105-12-77

29. Aran D, Hu Z, Butte AJ. xCell: digitally portraying the tissue cellular heterogeneity landscape. Genome Biol. (2017) 18:220. doi: 10.1186/s13059-017-1349-1

30. Feldman M, Corbett DB, Ramsey EJ, Walsh JH, Richardson CT. Abnormal gastric function in longstanding, insulin-dependent diabetic patients. Gastroenterology. (1979) 77:12-7. doi: 10.1016/S0016-5085(79)80003-7

31. Ramirez-Zamora S, Mendez-Rodriguez ML, Olguin-Martinez M, SanchezSevilla L, Quintana-Quintana M, Garcia-Garcia N, et al. Increased erythrocytes by-products of arginine catabolism are associated with hyperglycemia and could be involved in the pathogenesis of type 2 diabetes mellitus. PLoS ONE. (2013) 8:e66823. doi: 10.1371/journal.pone.0066823

32. Wake H, Ortiz FC, Woo DH, Lee PR, Angulo MC, Fields RD. Nonsynaptic junctions on myelinating glia promote preferential myelination of electrically active axons. Nat Commun. (2015) 6:7844. doi: 10.1038/ncomms8844

33. Chaves-Almagro C, Castan-Laurell I, Dray C, Knauf C, Valet P, Masri B. Apelin receptors: from signaling to antidiabetic strategy. Eur J Pharmacol. (2015) 763:149-59. doi: 10.1016/j.ejphar.2015.05.017

34. Zhou T, Guan H, Yao J, Xiong X, Ma A. The quality of life in Chinese population with chronic non-communicable diseases according to EQ-5D-3L: a systematic review. Qual Life Res. (2018) 27:2799814. doi: 10.1007/s11136-018-1928-y

35. Price CL, Knight SC. Advanced glycation: a novel outlook on atherosclerosis. Curr Pharm Des. (2007) 13:3681-7. doi: 10.2174/138161207783018608

36. Dong Y, Chen H, Gao J, Liu Y, Li J, Wang J. Molecular machinery and interplay of apoptosis and autophagy in coronary heart disease. J Mol Cell Cardiol. (2019) 136:27-41. doi: 10.1016/j.yjmcc.2019.09.001

37. Reiner Z, Guardamagna O, Nair D, Soran H, Hovingh K, Bertolini $\mathrm{S}$, et al. Lysosomal acid lipase deficiency-an under-recognized cause of dyslipidaemia and liver dysfunction. Atherosclerosis. (2014) 235:2130. doi: 10.1016/j.atherosclerosis.2014.04.003
38. Kidoya $\mathrm{H}$, Takakura N. Biology of the apelin-APJ axis in vascular formation. $J$ Biochem. (2012) 152:125-31. doi: 10.1093/jb/mvs071

39. Wang F, Fisher SA, Zhong J, Wu Y, Yang P. Superoxide dismutase 1 in vivo ameliorates maternal diabetes mellitus-induced apoptosis and heart defects through restoration of impaired wnt signaling. Circ Cardiovasc Genet. (2015) 8:665-76. doi: 10.1161/CIRCGENETICS.115. 001138

40. Zhang Z, Zhang HY, Zhang Y, Li $H$. Inactivation of the Ras/MAPK/PPARgamma signaling axis alleviates diabetic mellitus-induced erectile dysfunction through suppression of corpus cavernosal endothelial cell apoptosis by inhibiting HMGCS2 expression. Endocrine. (2019) 63:615-31. doi: 10.1007/s12020-018-1810-2

41. Medzikovic L, Schumacher CA, Verkerk AO, Van Deel ED, Wolswinkel R, Van Der Made I, et al. Orphan nuclear receptor Nur77 affects cardiomyocyte calcium homeostasis and adverse cardiac remodelling. Sci Rep. (2015) 5:15404. doi: 10.1038/srep15404

42. Wan X, Belanger K, Widen SG, Kuyumcu-Martinez MN, Garg NJ. Genes of the cGMP-PKG-Ca(2+) signaling pathway are alternatively spliced in cardiomyopathy: role of RBFOX2. Biochim Biophys Acta Mol Basis Dis. (2020) 1866:165620. doi: 10.1016/j.bbadis.2019. 165620

43. Gajate C, Alonso MT, Schimmang T, Mollinedo F. C-Fos is not essential for apoptosis. Biochem Biophys Res Commun. (1996) 218:26772. doi: 10.1006/bbrc.1996.0047

44. Kermani ES, Nazari Z, Mehdizadeh M, Shahbazi M, Golalipour MJ. Gestational diabetes influences the expression of hypertrophic genes in left ventricle of rat's offspring. Iran J Basic Med Sci. (2018) 21:5258. doi: 10.22038/IJBMS.2018.25116.6233

45. Florian M, Jankowski M, Gutkowska J. Oxytocin increases glucose uptake in neonatal rat cardiomyocytes. Endocrinology. (2010) 151:48291. doi: 10.1210/en.2009-0624

46. Watanabe S, Wei FY, Matsunaga T, Matsunaga N, Kaitsuka T, Tomizawa K. Oxytocin protects against stress-induced cell death in murine pancreatic beta-cells. Sci Rep. (2016) 6:25185. doi: 10.1038/ srep25185

47. Klement J, Ott V, Rapp K, Brede S, Piccinini F, Cobelli C, et al. Oxytocin improves beta-cell responsivity and glucose tolerance in healthy men. Diabetes. (2017) 66:264-71. doi: 10.2337/db16-0569

Conflict of Interest: The authors declare that the research was conducted in the absence of any commercial or financial relationships that could be construed as a potential conflict of interest.

Copyright (c) 2020 Huang, Deng, Wei, Wang, Zou and Wei. This is an open-access article distributed under the terms of the Creative Commons Attribution License (CC $B Y)$. The use, distribution or reproduction in other forums is permitted, provided the original author(s) and the copyright owner(s) are credited and that the original publication in this journal is cited, in accordance with accepted academic practice. No use, distribution or reproduction is permitted which does not comply with these terms. 